\title{
Atividade antagonista do óleo essencial de Lippia alba (Mill.) N. E. Brown (Verbenaceae) sobre Meloidogyne incognita (Kofoid \& White) Chitwood
}

GONÇALVES, F.J.T..*; BARBOSA, F.G.2; LIMA, J.S.2; COUTINHO, I.B.L.2; OLIVEIRA, F.C.2; ROCHA, R.R.2; ANDRADE NETO, M. ${ }^{2}$

1Universidade Federal Rural de Pernambuco (UFRPE), Rua Dom Manoel de Medeiros, s/n, Dois Irmãos, CEP: 52171-900, Recife - PE. 2Universidade Federal do Ceará (UFC), Av. Mister Hull, s/n, Campus do Pici, CEP: 60.455-970, Fortaleza - CE. *Autorpara correspondência: goncalvesfj@yahoo.com.br

RESUMO: O controle de fitonematóides é uma tarefa difícil. A alta infestação de nematoides no solo obriga os produtores a usarem doses mais elevadas de nematicidas, ou ainda, a aumentarem a freqüência das aplicações, geralmente no solo, ocasionando maior potencial de dano ao homem e ao ambiente. Além disso, nematicidas convencionais vêm sofrendo grandes restrições de uso em muitos países. Desse modo, a busca de novas medidas de controle de fitonematóides é uma prioridade da agricultura sustentável. Desta forma, objetivou-se estudar o efeito de óleos essenciais de Lippia Alba, na mortalidade de juvenis pré-parasitas do segundo estádio de Meloidogyne incognita. O ensaio foi montado em delineamento inteiramente casualizado, com 4 repetições, em esquema fatorial $3 \times 4$ ( 3 quimiotipos $\times 4$ concentrações) empregando-se três diferentes quimiotipos de L. Alba (I, II, III) nas concentrações de 0, 100, 500 e 1000 ppm, coletados em diferentes horas do dia (7, 9, 13, 16 e 19h). Os dados coletados (\% de mortalidade) foram submetidos à análise de variância e os valores médios comparados por meio do teste de Tukey. Foi possível concluir que Lippia alba contém, em seu óleo essencial, compostos com efeitos significativos na mortalidade de juvenis de segundo estádio (J2) de M. incognita. Dessa forma, é importante destacar que o acentuado efeito nematicida do óleo essencial de $L$. alba sugere a possibilidade de seu uso no controle de $M$. incognita.

Palavras-chave: Lippia Alba. Quimiotipo. Nematóide.

ABSTRACT: Antagonist activity of the essential oil Lippia alba (Mill.) N. E. Brown (Verbenaceae) on Meloidogyne incognita (Kofoid \& White) Chitwood. The control of nematodes is difficult. The high infestation of nematodes in the soil requires the producers use higher doses of nematicides, increasing the frequency of applications, usually in soil, causing negative effects to humans and the environment. Furthermore, conventional nematicides have suffered great use restrictions in many countries. Thus, the search for new measures to control nematodes is a priority of sustainable agriculture. Thus, the objective of this study was available the effect of essential oils from Lippia Alba against Meloidogyne incognita. The experiment was conducted in a completely randomized design with four replications in a factorial $3 \times 4$ ( 3 chemotypes and 4 concentrations) using three different chemotypes of $L$. Alba (I, II, III) at concentrations of $0,100,500$ and $1000 \mathrm{ppm}$, collected in different times of day (7 AM, 9 AM, $1 \mathrm{PM}, 4 \mathrm{PM}$ e $7 \mathrm{PM}$ ). The data collected (\% mortality) were subjected to analysis of variance and mean values were compared using the Tukey test. It was able to conclude that $L$. alba contains in its essential oil, compounds with significant effects on mortality of $M$. incognita. Thus, it is important to note that the greatest nematicide effect of essential oil of $L$. alba suggests the possibility of its use to control $M$. incognita.

Keywords: Lippia Alba. Chemotype. Nematode. 


\section{INTRODUÇÃO}

Os nematóides são organismos alongados e afilados em ambas as extremidades, encontrados desde regiões extremamente frias até regiões de desertos, distribuídos em mais de 500.000 espécies (Tsai et al., 1991). De acordo com o autor supracitado, os nematóides são classificados em três grupos: parasitas de animais; de vida livre, habitando tanto o solo como águas doces e salgadas; e parasitas de plantas, conhecidos como fitonematóides. Estes últimos causam enormes danos a diversas culturas, ocasionando grandes perdas na produção agrícola (Campos, 1992), sendo classificados em ectoparasitas migradores, semi-endoparasitas migradores e endoparasitas (McDonald, 1979).

Fitonematóides do gênero Meloidogyne são os que causam maiores prejuízos, o que se deve à sua capacidade de adaptação aos diversos agroecossistemas, sendo considerados de difícil controle, causando danos drásticos a horticultura e a campos com grandes culturas (Andrés et al., 2012), causando perdas estimadas em bilhões de euros anualmente (Bleve-Zacheo et al., 2007) com redução de 50 a até $90 \%$ na produção das culturas afetadas (Echeverrigaray et al., 2010; Marino et al., 2012; Mullin et al., 1991).

O controle de fitonematóides é uma tarefa difícil, principalmente em plantas perenes. Várias estratégias são utilizadas para seu controle e todas elas apresentam certo grau de eficiência quando utilizadas dentro de um plano de manejo integrado e racional. Dentre os métodos utilizados estão: uso de cultivares resistentes, rotação de culturas, sendo de difícil manejo devido à ampla gama de hospedeiros que esse patógeno ataca e, o controle químico que, quando utilizando em excesso e de forma desequilibrada, há o acúmulo de resíduos tóxicos nos alimentos, intoxicações de seres vivos, a contaminação do solo e da água, a eliminação de inimigos naturais, o desequilíbrio biológico e a resistência de pragas e doenças a produtos químicos (Damasceno et al., 2015; Lordello, 1984; Marino et al., 2012; Moreira et al., 2009), o que tem ensejado a pesquisa sobre o uso de moléculas menos danosas ao homem e ao meio ambiente. No entanto, devido à facilidade na aquisição desses insumos e, nos resultados de controle de doenças em curto prazo, observa-se uma grande utilização de produtos sintéticos, visando controlar doenças em plantas (Ferreira et al., 2012a).

A busca por novos métodos de controle para doenças de plantas é intensa em virtude da crescente resistência dos microrganismos patogênicos aos produtos sintéticos (Cruz et al., 2012a). No entanto, frente à preocupação com os danos que esses produtos causam, há um aumento na demanda por pesquisas que visam o controle alternativo de nematóides, onde relatam que os óleos essenciais extraídos de algumas espécies de plantas, são eficientes em inibir a atividade nematicida e podem ser utilizadas como uma alternativa ecologicamente benéfica ao controle deste patógeno (Bruni et al., 2004; Marino et al., 2012; Moreira et al., 2009).

Os óleos essenciais extraídos de plantas podem ser empregados na obtenção de novos nematicidas, uma vez que alguns compostos encontrados nestes apresentam potencial atividade biológica e química (Lee et al., 2001), ressaltando que a composição destes são passíveis de serem influenciados por fatores externos como por exemplo o horário de colheita (Echeverrigaray et al., 2010; Ehlert et al., 2013). Estudos com óleos essenciais no controle de nematóides ainda são pouco expressivos (Oka et al., 2000), embora esses produtos não causem danos ao ambiente (Isman, 2000). Segundo Silveira et al. (2012), é crescente o mercado e o número de pesquisas com óleos essenciais, mostrando seus efeitos antimicrobianos e suas inúmeras aplicações.

Os constituintes químicos de óleos essenciais podem pertencer às mais diversas classes de substâncias. De acordo com Marino et al. (2012), os óleos essenciais são compostos voláteis resultantes do metabolismo secundário das plantas, cujos principais componentes são os terpenos e terpenóides. O mecanismo da ação nematicida dos óleos essenciais ou de seus constituintes ainda não está bem claro, no entanto sugere-se que os componentes do óleo essencial podem agir no sistema nervoso, afetando o comportamento, desenvolvimento e outros processos controlados por enzimas nos nematóides (Oka, 2001). Outra possibilidade é que os óleos essenciais rompam a membrana celular do nematóide e alterem a sua permeabilidade.

Vários autores relatam o efeito nematicida de óleos essenciais de diferentes espécies vegetais. De acordo com Sangwan et al. (1985), os constituintes químicos do óleo essencial de gramíneas do gênero Cymbopogon, quando combinados, exercem importante papel na atividade nematicida. O óleo essencial de Pelargonium graveolens demonstrou atividade nematicida a Meloidogyne incognita, em decorrência da presença de substâncias químicas como o citronelol, geraniol e linalol (Leela et al., 1992). O furfural, substância encontrada em muitos óleos essenciais de plantas, também apresenta ação nematicida a $M$. incognita, M. arenaria, Pratylenchus brachyurus e Heterodera glycines (Rodriguez-Kabana et al., 1993). Marino et al. (2012) observaram que o óleo de Lippia alba

Rev. Bras. PI. Med., Campinas, v.18, n.1, p.157-167, 2016. 
apresentou efeito nematicida in vitro, reduzindo a taxa de eclosão de $M$. incognita raça 1 e favorecendo a mortalidade do estádio juvenil J2 eclodido.

O óleo essencial de Lippia alba é constituído por sesquiterpenos e monoterpenos, monocíclicos ou acíclicos, com variações qualitativas e quantitativas dos teores de carvona, limoneno, mirceno, linolol, neral e geranial (Aguiar \& Costa, 2005), sendo que os compostos a-pineno, trans-cariofileno, germacreno D-4-ol não são influenciados pelos horários de colheita uma vez que o tricicleno estava presente somente às $8 \mathrm{~h}$ e o bulnesol às $8 \mathrm{~h}$ e às $10 \mathrm{~h}$ (Ehlert et al., 2013). Diversas outras espécies de plantas produzem óleos essenciais que apresentaram efeito nematicida (Andrés et al., 2012; Bakkall et al., 2008; Freitas et al., 2000; Moreira \& Ferreira, 2015).

A necessidade de se obter sustentabilidade ambiental tem gerado a necessidade cada vez maior, de se testar produtos naturais, visando um controle alternativo de fitopatógenos (Cruz et al., 2012a). Embora ocorra no Brasil uma enorme diversidade de espécies de plantas, os estudos sobre o efeito de suas substâncias produzidas ainda são escassos. Desta forma, objetivou-se estudar, in vitro, o efeito de óleos essenciais de Lippia Alba, na mortalidade de juvenis pré-parasitas do segundo estádio (J2) de M. incognita.

\section{MATERIAL E MÉTODOS Obtenção de juvenis de segundo estádio} (J2)

A partir de cultura pura de M. incognita, mantida em Vernonia sp., em casa-de-vegetação, obtiveram-se os juvenis (J2). As plantas inoculadas foram cultivadas em vasos de $600 \mathrm{~mL}$, tendo como substrato areia mais vitassolo, na proporção de 3:1 (v/v), respectivamente. Após 45 dias, os sistemas radiculares de Vernonia sp., infestados, foram separados da parte aérea e lavados cuidadosamente em um recipiente, evitando agitação para não haver desprendimento das massas de ovos aderidas. Em seguida, as raízes foram levadas ao laboratório, onde as massas de ovos foram extraídas, de forma manual, com o auxílio de dois estiletes metálicos e um microscópio estereoscópico. Depois de extraídas, as massas de ovos foram colocadas em uma placa de Petri com água destilada. Efetuou-se a extração em, aproximadamente, duas horas, e as placas de Petri contendo as massas de ovos foram mantidas à temperatura de $25^{\circ} \mathrm{C}$ durante 24 horas para que houvesse a eclosão dos juvenis (J2).

\section{essenciais \\ Extração e caracterização dos óleos}

Para a extração dos óleos essenciais, empregou-se a técnica de "arraste com vapor d'água" (Simões \& Spitzer, 1999). Das folhas de $L$. alba, extraiu-se os óleos essenciais quimiotipos I, II e III. Meio quilo das folhas, coletadas em diferentes horários do dia $(7,9,13,16,19 \mathrm{~h})$, foram picadas, pesadas e colocadas em um balão de fundo redondo e contendo dois litros de água. No aparelho de Clavenger realizou-se a extração dos óleos das folhas por meio do vapor d'água, que passava através de um tubo que ligava o balão com água em ebulição, por um aquecedor interno, ao balão com as folhas. Os componentes vegetais extraídos pelo arraste em vapor d'água foram coletados na forma de hidrolato em um frasco de vidro após a passagem por um condensador tipo Liebig. O processo de extração ocorreu em aproximadamente 3 horas quando, então, as folhas apresentaram coloração amarelada. Ao hidrolato obtido adicionou-se sulfato de sódio anidro para a secagem do óleo. Os óleos puros foram acondicionados em uma geladeira a $10^{\circ} \mathrm{C}$, até o momento de sua utilização, sendo também submetidos a análises químicas, através de cromatografia gasosa acoplada a um espectrômetro de massa.

\section{Preparo dos óleos essenciais para os testes de mortalidade}

Para a solubilização dos óleos preparou-se uma solução utilizando $2 \mathrm{~mL}$ de Dimetil sulfóxido para $98 \mathrm{~mL}$ de água destilada, obtendo-se uma solução final de DMSO ( $2 \%$ v/v em água). Após as pesagens dos óleos e adição das soluções obtiveram-se as concentrações de 0, 100, 500 e 1000 ppm.

\section{Efeito dos óleos essenciais sobre juvenis de segundo estádio (J2)}

Em vidros nematológicos, colocou-se $1 \mathrm{~mL}$ do óleo essencial preparado como descrito anteriormente. Empregaram-se os óleos de L. alba, quimiotipos I, II e III, denominados em função das diferenças no constituinte majoritário de seus óleos essenciais: citral, carvona e linalol, respectivamente (Julião et al., 2003). Desse modo, cada vidro recebeu $100 \mathrm{~J} 2$ de M. incognita, que foram retirados da placa de Petri com o auxílio de uma pipeta graduada para $50 \mu \mathrm{L}$. A seguir, os vidros foram colocados em placas maiores para posterior incubação.

A percentagem de (J2) mortos foi avaliada após mais $24 \mathrm{~h}$ de incubação. Foram caracterizados como mortos aqueles $\mathrm{J} 2$ que permaneceram com o corpo completamente distendido após este período.

$\mathrm{O}$ ensaio foi montado em delineamento inteiramente casualizado (DIC), com 4 repetições, em esquema fatorial $3 \times 4$ ( 3 quimiotipos $\times 4$ concentrações) empregando-se três diferentes quimiotipos de L. Alba (quimiotipo I, II e III), coletados em diferentes horários do dia, nas concentrações de 0, 100, 500 e 1000 ppm. 
Os dados coletados (\% de mortalidade) foram submetidos à análise de variância, utilizando o programa estatístico Sisvar 5.3 (Ferreira, 2008). Os valores médios referentes ao percentual de mortalidade dos juvenis (J2) de M. incognita foram comparadas por meio do teste de Tukey, ao nível de $5 \%$ de significância.

\section{RESULTADOS E DISCUSSÃO}

Todos os quimiotipos testados proporcionaram mortalidade significativa quando comparados com a testemunha (Tabela 1). Segundo Oka (2000), a atividade nematicida de óleos essenciais se deve à presença de monoterpenos oxigenados, como constituintes químicos majoritários. Estudado a atividade nematicida de Carum carvi, Foenicum vulgare, Mentha rotundifolia, Mentha spicata, Origanum vulgare, O. syriacum e Coridothymus capitatus, o autor supracitado concluiu que essa ação se devia a presença de compostos oxigenados como, carvacrol, E-anetol, timol e carvona. O óleo essencial de L. alba (Tabela 2) apresenta constituição química semelhante aos óleos investigados por Oka (2000), daí sua excelente ação observada neste estudo.

A concentração de 500 ppm foi tão eficiente na ação nematicida quanto a concentração de 1000 ppm de todos os três quimiotipos de óleo essencial, não diferindo entre si em relação ao efeito nematicida, medido pelo percentual de mortalidade causados em juvenis (J2) de $M$. incognita, apresentando mortalidade superior a $96 \%$. Resultados semelhantes foram encontrados por Marino et al. (2012). Igualmente como observado por estes autores, as concentrações testadas provocaram a morte de juvenis (J2) de M. incognita de forma expressiva, indicando haver uma ação tóxica do óleo testado sobre o fitopatógeno. Segundo Marino et al. (2012), os óleos essenciais podem interagir com a membrana citoplasmática promovendo a ruptura da estrutura de polissacarídeos, lipídeos e fosfolipídios, ocasionando a despolarização das membranas das organelas citoplasmáticas, como as mitocôndrias, resultando na liberação de íons de cálcio e proteínas. Além disso, também podem alterar a permeabilidade das membranas. De acordo com os autores supracitados, estes mecanismos de ação poderiam explicar a elevada mortalidade dos juvenis (J2) de $M$. incognita, na presença do óleo de L. alba.

Resultados promissores utilizando óleos essenciais de plantas no controle de fitopatógenos têm sido relatados na literatura. Cruz et al. (2012b) obtiveram excelentes resultados utilizando óleo essencial de hortelã pimenta sobre Fusarium solani. Nos seus resultados, foi possível observar que, mesmo em baixa concentração do óleo, ocorre uma considerável inibição do crescimento micelial do fungo e, concentrações acima de $15 \mu \mathrm{L} . \mathrm{mL}^{-1}$ são responsáveis pela inibição total do crescimento micelial do fitopatógeno. Ferreira et al. (2012b) também obtiveram satisfatórios utilizando óleos essenciais para o controle de Colletotrichum gloeosporioides.

A atividade nematicida do óleo essencial de $L$ alba mostra a possibilidade do uso deste composto no controle de fitonematóides, uma vez que, os nematicidas convencionais são caros, altamente tóxicos, persistentes de amplo espectro de ação (Ferraz, 1997). De acordo com Silva et al. (2003), a atividade de óleos essenciais sobre fitopatógenos tem relação com sua propriedade hidrofóbica, uma vez que em contato organismos patogênicos causa distúrbios fisiológicos a nível celular, alterando a permeabilidade das membranas celulares e expondo suas organela às intempéries do meio.

A composição do óleo essencial de $L$. alba apresenta variação quantitativa e qualitativa, levando à separação em quimiotipos (Julião et al., 2003; Tavares et al., 2005). Os quimiotipos produtores de citral, carvona e linalol, foram denominados $L$. alba 1,2 e 3 , respectivamente.

O horário de coleta do óleo essencial de

TABELA 1. Percentual médio de mortalidade de juvenis (J2) de $M$. incognita, submetidos a diferentes concentrações de três quimiotipos de óleos essenciais de $L$. Alba.

\begin{tabular}{lcccc}
\hline \multirow{2}{*}{ Tratamentos } & \multicolumn{3}{c}{ Quimiotipos } & Média \\
\cline { 2 - 4 } & Quimiotipo I & Quimiotipo II & Quimiotipo III & $0,0 \mathrm{a}$ \\
\hline Testemunha & $0,0 \mathrm{aA}$ & $0,0 \mathrm{aA}$ & $0,0 \mathrm{aA}$ & $23,7 \mathrm{~b}$ \\
$100 \mathrm{ppm}$ & $21,9 \mathrm{bB}$ & $24,8 \mathrm{bA}$ & $24,5 \mathrm{bA}$ & $98,0 \mathrm{c}$ \\
$500 \mathrm{ppm}$ & $96,9 \mathrm{cB}$ & $99,8 \mathrm{cA}$ & $97,2 \mathrm{cB}$ & $99,6 \mathrm{c}$ \\
$1000 \mathrm{ppm}$ & $99,7 \mathrm{cA}$ & $99,1 \mathrm{cA}$ & $100,0 \mathrm{cA}$ & \\
\hline Média & $54,6 \mathrm{~A}$ & $55,9 \mathrm{~A}$ & $55,4 \mathrm{~A}$ & \\
\hline
\end{tabular}

Médias seguidas pela mesma letra minúscula e maiúscula, nas colunas e linhas, respectivamente, não diferem entre si pelo teste de Tukey ao nível de $5 \%$ de significância.

Rev. Bras. Pl. Med., Campinas, v.18, n.1, p.149-156, 2016. 
TABELA 2. Compostos, Índice de Kováts e composição química (\%) dos três quimiotipos dos óleos essências das folhas de L. Alba.

\begin{tabular}{|c|c|c|c|c|c|}
\hline \multirow{2}{*}{$\mathbf{N}^{\circ}$} & \multirow{2}{*}{ Composto } & \multirow{2}{*}{ IK } & \multicolumn{3}{|c|}{ Percentagem (\%) } \\
\hline & & & Quimiotipo I & Quimiotipo II & Quimiotipo III \\
\hline 1 & Sabineno & 975 & & $0,77 \pm 0,07$ & $5,07 \pm 0,30$ \\
\hline 2 & Mirceno & 988 & $4,90 \pm 0,37$ & $1,35 \pm 0,12$ & $0,39 \pm 0,16$ \\
\hline 3 & $\rho$-Cimeno & 1024 & $0,432 \pm 0,27$ & $2,45 \pm 0,29$ & \\
\hline 4 & Limonemo & 1029 & & $9,29 \pm 0,89$ & $16,31 \pm 1,14$ \\
\hline 5 & (E)- $\beta$-Ocimeno & 1050 & & & $0,12 \pm 0,09$ \\
\hline 6 & ү-Terpineno & 1059 & $1,09 \pm 0,12$ & $3,75 \pm 0,29$ & $1,58 \pm 0,03$ \\
\hline 7 & cis-Crisantenol & 1070 & & & $0,58 \pm 0,43$ \\
\hline 8 & Linalol & 1096 & $0,60 \pm 0,03$ & & \\
\hline 9 & cis-Diidrocarveol & & $0,73 \pm 0,10$ & & \\
\hline 10 & Iso-Diidrocarveol & & $1,25 \pm 0,46$ & $1,23 \pm 0,32$ & \\
\hline 11 & Trans-Carveol & 1216 & & $0,27 \pm 0,24$ & \\
\hline 12 & B-Citronelol & 1225 & $0,69 \pm 0,52$ & & \\
\hline 13 & Neral & 1238 & $22,49 \pm 0,79$ & $28,29 \pm 0,89$ & \\
\hline 14 & Carvona & 1243 & & & $62,30 \pm 1,09$ \\
\hline 15 & Piperitona & 1252 & & & $0,26 \pm 0,16$ \\
\hline 16 & Geranial & 1267 & $34,64 \pm 1,74$ & $38,88 \pm 1,42$ & \\
\hline 17 & a-Copaeno & 1376 & $2,05 \pm 0,39$ & & \\
\hline 18 & $\beta$-Cubebeno & 1388 & $0,33 \pm 0,18$ & $0,30 \pm 0,12$ & \\
\hline 19 & $\beta$-Lemeno & 1390 & $2,48 \pm 0,24$ & & \\
\hline 20 & $\beta$-Cariofileno & 1419 & $6,31 \pm 0,75$ & $6,22 \pm 0,82$ & \\
\hline 21 & a-Cubebeno & 1348 & & $2,74 \pm 0,72$ & \\
\hline 22 & Piperitona & 1349 & & & $1,44 \pm 0,09$ \\
\hline 23 & Ciclosativeno & 1371 & & $0,28 \pm 0,26$ & \\
\hline 24 & a-Humuleno & 1454 & $0,58 \pm 0,33$ & & \\
\hline 25 & allo-Aromadreno & 1460 & & $0,26 \pm 0,14$ & $3,41 \pm 0,60$ \\
\hline 26 & y-Muuroleno & 1479 & $3,00 \pm 0,32$ & & \\
\hline 27 & $\alpha-S e l i n e n o$ & 1498 & $0,52 \pm 0,03$ & & \\
\hline 28 & (Z)- y-Bisaboleno & 1515 & & $0,89 \pm 0,40$ & \\
\hline 29 & $\beta$-Sesquifelandreno & 1522 & & & $5,81 \pm 0,58$ \\
\hline 30 & $\delta$-Cardineno & 1523 & $0,84 \pm 0,09$ & & \\
\hline 31 & (E)- y-Bisaboleno & 1531 & & & $0,12 \pm 0,08$ \\
\hline 32 & Elemol & 1549 & $11,56 \pm 0,37$ & & \\
\hline 33 & Óxido de cariofileno & 1583 & $1,04 \pm 0,27$ & & \\
\hline 34 & Guaiol & 1600 & $0,56 \pm 0,03$ & & \\
\hline 35 & a-Eudesmol & 1653 & $1,40 \pm 0,07$ & & \\
\hline Total & & & 97,49 & 96,96 & 99,15 \\
\hline
\end{tabular}

L. alba quimiotipo I não influencia na sua ação contra fitonematóides (Figura 1), uma vez que a ação desse quimiotipo apresentou o mesmo efeito nematicida, independente do horário de coleta, pois apresentava composição química semelhante, como demonstrado nos pequenos valores de desvio padrão (Tabela 2), esta atividade pode estar relacionada a presença dos compostos mirceno, linalol, $\beta$-citronelol, $\alpha$-eudesmol, que apresentaram atividade nematicida (Echeverrigaray et al., 2010) e, principalmmente, neral e geranial, que mostraramse muito ativos para nematóides Bursaphelenchus xylophilus (Park et al., 2007).

De acordo com Matos (1996), o quimiotipo I é produtor de óleos essenciais ricos em mirceno, neural e geranial. Dessa forma é possível concluir que esses compostos não são influenciados por condições de clima durante sua extração, como pode ser observado nos pequenos valores de desvio padrão para os compostos (Tabela 2).

A ação nematicida do óleo essencial de L. alba quimiotipo II apresentou variação dependendo do horário de coleta (Figura 2). Em baixas concentrações, esse mostrou melhor efeito 


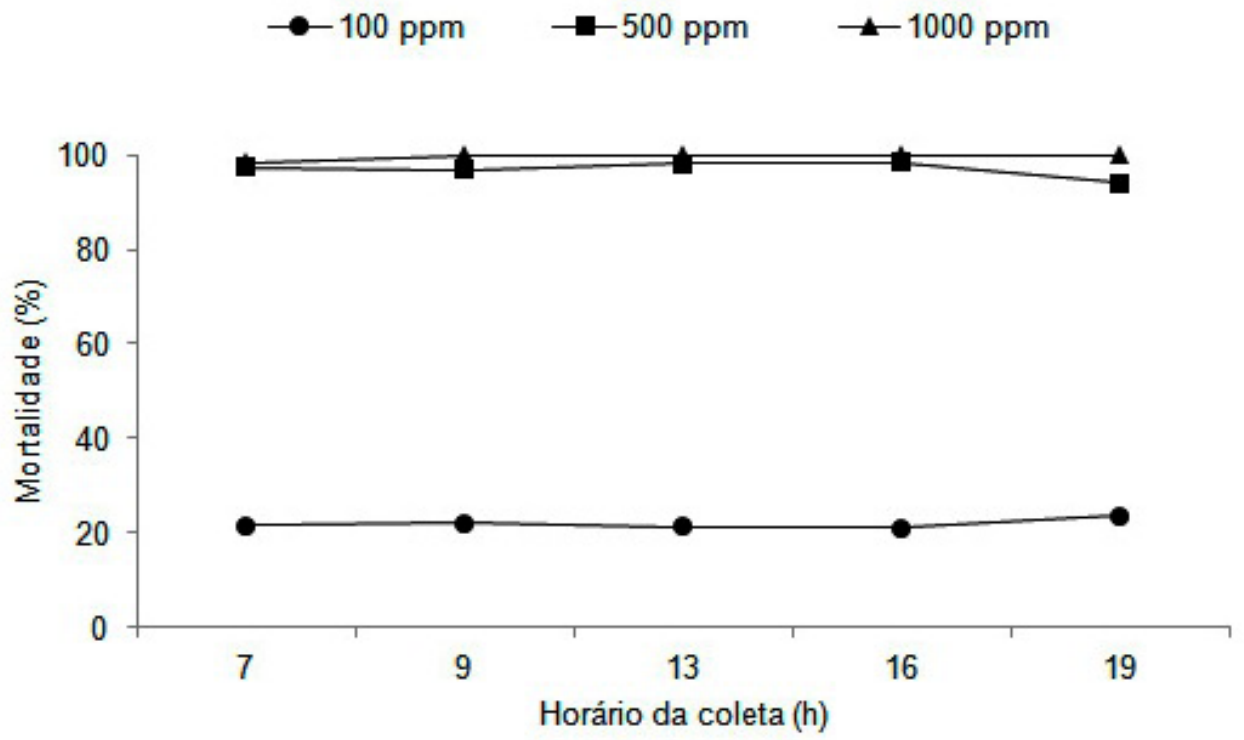

FIGURA 1. Percentual médio de mortalidade de juvenis do segundo estágio (J2) de M. incognita submetidos a diferentes concentrações do óleo de $L$. alba quimiotipo I, coletado em diferentes horários do dia.

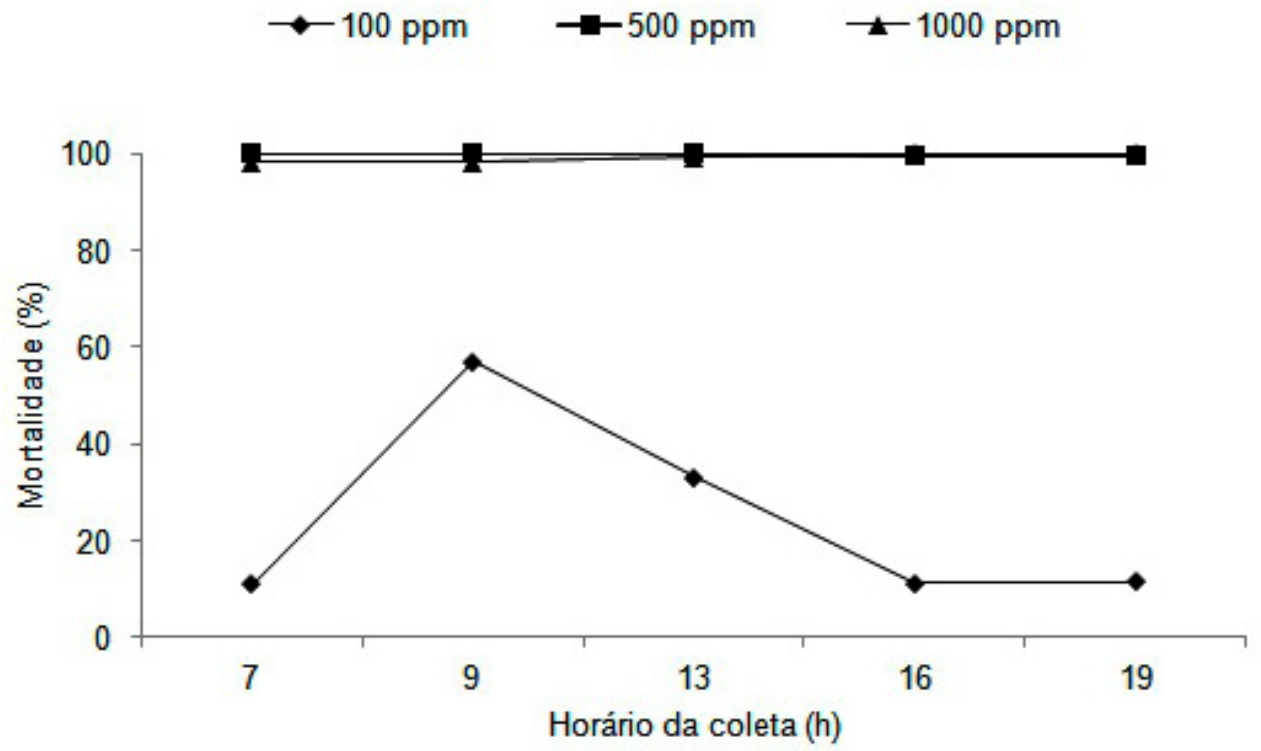

FIGURA 2. Percentual médio de mortalidade de juvenis do segundo estágio (J2) de M. incognita submetidos a diferentes concentrações do óleo de $L$. alba quimiotipo II, coletado em diferentes horários do dia.

nematicida quando coletado nas primeiras horas da manhã. Segundo Matos (1996), o quimiotipo II do óleo essência de $L$. alba apresenta limoneno, neral e geranial como componentes principais.

As horas mais quentes do dia comprometem a coleta de óleo essencial de $L$. alba quimiotipo III, quando em concentrações baixas, para ser usado como nematicida (Figura 3). Esse quimiotipo, quando coletado por vota do meio dia, ocasional menor mortalidade de juvenis do segundo estágio (J2) de Meloidogyne incognita. Esta atividade pode estar relacionada a presença dos compostos mirceno, linalol e carvona (Echeverrigaray et al., 2010; Ntalli et al., 2011). Atividades nematicidas dessa espécie vegetal já foram investigadas e comprovadas em estudos anteriores (Moreira et al., 2009; Marino et al., 2012; Damasceno et al., 2015).

O quimiotipo III do óleo essencial de $L$. alba tem limoneno e carvona como componentes principais. Daí a classificação do quimiotipo III como quimiotipo limoneno-carvona para uma população que se encontre sobre as mesmas condições edafoclimáticas (Matos, 1996).

Quando utilizados em concentração acima de 500 ppm, os três quimiotipos de $L$. alba não mostraram variação em sua ação nematicida, independente do horário de coleta pois apresentava composição química semelhante, como demonstrado 


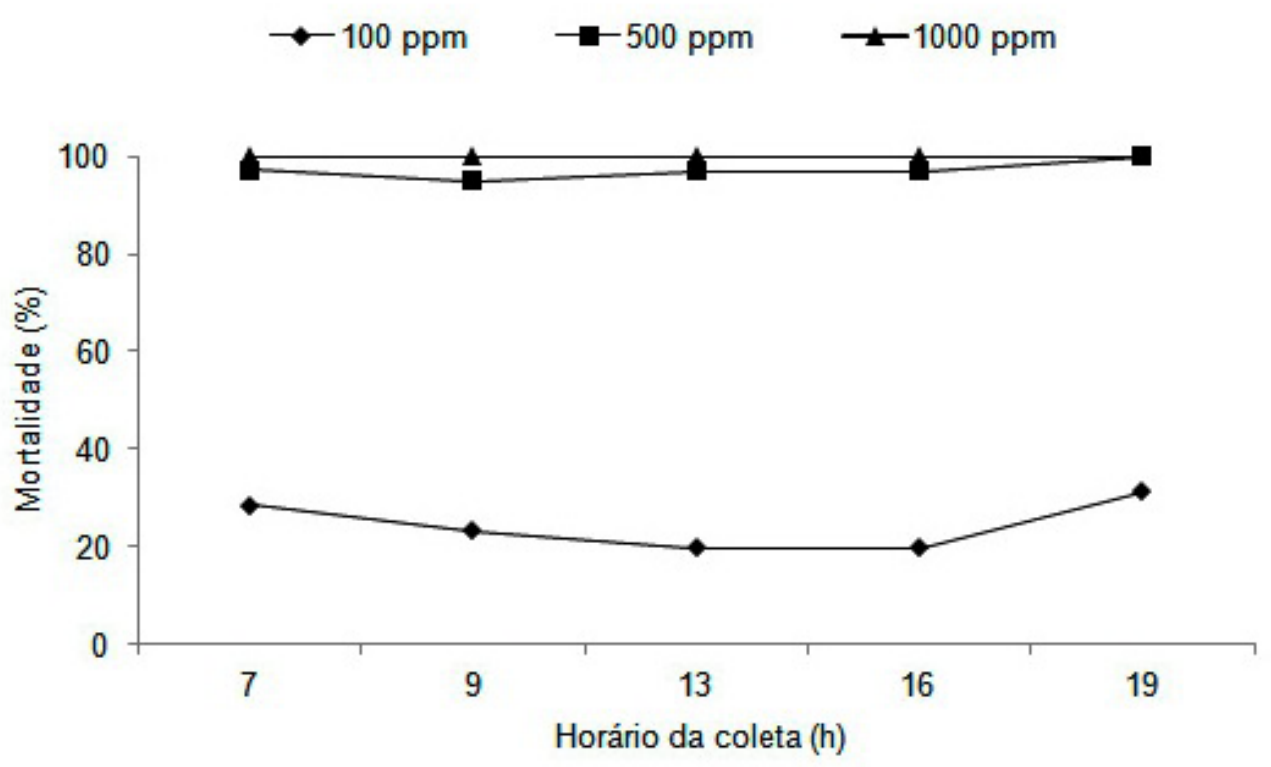

FIGURA 3. Percentual médio de mortalidade de juvenis do segundo estágio (J2) de M. incognita submetidos a diferentes concentrações do óleo de $L$. alba quimiotipo III, coletado em diferentes horários do dia.

nos pequenos valores de desvio padrão (Tabela 2).

Pode-se observar que Lippia alba contém, em seu óleo essencial, compostos com efeitos significativos na mortalidade de juvenis de segundo estádio (J2) de $M$. incognita. Os três quimiotipos do óleo essencial de $L$. alba apresentam efeito semelhante na concentração de 1000 ppm, ocasionando mortalidade acima de $99 \%$ de juvenis de segundo estádio (J2) de M. incognita.

A atividade nematicida do óleo essencial de $L$. alba quimiotipo I, não apresentou diferença na mortalidade dos juvenis de segundo estádio (J2) de $M$. incognita, em função do horário de coleta, enquanto os quimiotipos II e III, apresentaram diferenças nesta variável em função do horário de coleta. Diante disto, sugere-se que o óleo essencial de $L$. alba possui um acentuado efeito nematicida no controle de $M$. incognita.

\section{AGRADECIMENTOS}

Agradecemos ao Conselho Nacional de Pesquisa (CNPq) pela concessão de bolsa e a Universidade Federal do Ceará (UFC) pela infraestrutura.

\section{REFERÊNCIAS}

ANDRÉS, M.F. et al. Nematicidal activity of essential oils: a review. Phytochemistry Reviews, v.11, n.4, p.371390, 2012.

AGUIAR, J.S.; COSTA, M.C.C.D. Lippia alba (Mill.) N. E. Brown (Verbenaceae): levantamento de publicações nas áreas química, agronômica e farmacológica, no período de 1979 a 2004. Revista Brasileira de Plantas
Medicinais, v.8, n.1, p.79-84, 2005.

BAKKALI, F. et al. Biological effects of essential oils-A review. Food and chemical toxicology, v.46, n.4, p.446-475, 2008.

BLEVE-ZACHEO, T. et al. The contribution of biotechnology to root-knot nematode control in tomato plants. Pest Tech, v.1, p.1-16, 2007.

BRUNI, R. et al. Chemical composition and biological activities of Ishpingo essential oil, a traditional Ecuadorian spice from Ocotea quixos (Lam.) Kosterm. (Lauraceae) flower calices. Food Chemical, v.85, n.4, p.415-421, 2004.

CAMPOS, P. Implicação da sobrevivência dos nematoides em solo e raízes de plantas no controle de fitopatógenos. Informe Agropecuário, n.16, p.15-16, 1992.

CRUZ, T.P. et al. Avaliação da atividade de extratos aquosos de canela, cravo e alho sobre Fusarium oxysporum f. sp. zingiberi. Enciclopédia Biosfera, v.8, n.15, p.237-247, 2012a.

CRUZ, T.P. et al. Composição química e avaliação do potencial fungicida do óleo essencial de Plectranthus amboinicus sobre Fusarium solani UENF/163 da goiabeira. Enciclopédia Biosfera, v.8, n.15, p. 466478, 2012b.

DAMASCENO, J.C.A. et al. Sisal leaf decortication liquid residue for controlling Meloidogyne javanica in tomato plants. Horticultura Brasileira, v.33, n.2, p.155-162, 2015.

ECHEVERRIGARAY, S. et al. Nematicidal Activity of Monoterpenoids Against the Root-Knot Nematode Meloidogyne incógnita. Nematology, v.100, n.2, 2010.

EHLERT, P.A.D. et al. Influência do horário de colheita sobre o rendimento e composição do óleo essencial de erva-cidreira brasileira [Lippia alba (Mill.) N. E. Br.] Revista brasileira de Plantas Medicinais, v.15, n.1, p.72-77, 2013.

FERRAZ, S. Evolução e situação atual dos problemas nematológicos das regiões Centro-oeste, Sudeste e Sul do Brasil. In: Congresso Brasileiro de Nematologia, 
20. Gramado. Gramado: SBN/UFP, p.3-4, 1997.

FERREIRA, D.F. SISVAR - Sistema de análise de variância para dados balanceados: programa de análises estatísticas e planejamento de experimentos Versão 5.3. Lavras: DEX/UFLA, 2008. Software.

FERREIRA, J.B. et al. Efeito da temperatura e óleos essenciais sobre o crescimento micelial de Fusarium solani isolado de mudas de Euterpe oleracea Mart (açaí). Enciclopédia Biosfera, v.8, n.14, p. 453-465, 2012a.

FERREIRA, J.B. et al. Óleos essenciais no controle de Colletotrichum gloeosporioides, agente causal da antracnose em palmáceas. Enciclopédia Biosfera, v.8, n.14, p.751-760, 2012b.

FREITAS, L.G. et al. Controle de Meloidogyne javanica com aplicação de óleos essenciais de pimenta malagueta (Capsicum frutescens) e mostarda (Brassica campestris). Fitopatologia brasileira, suplemento, Brasília, v.25, p.336, 2000.

ISMAN, M.B. Plant essencial oils for pest and disease management. Crop Protection, v.19, p.603-608, 2000.

JULIÃO, L.S. et al. Cromatografia em camada fina de extratos de três quimiotipos de Lippia alba (Mill) N.E.Br. (erva-cidreira). Revista Brasileira de Famacognosia, v.13, suplemento, p.36-38, 2003.

LEELA, N.K. et al. Nematicidal activity of essential oil of Pelargonium graveolens against the root-knot nematode Meloidogyne incognita. Nematologia Mediterranea, v.20, p.57-58, 1992.

LEE, M.R. et al. An assessment of the potential use of the nematode to copepod ratio in the monitoring of metals pollution: The Chañaral case. Marine Pollution Bulletin, n.42, p.696-701, 2001.

LORDELLO, L.G.E. Nematóides de plantas cultivadas. 8.ed. São Paulo: Nobel, 1984. 314 p.

MARINO, R.H. et al. Controle de Meloidogyne incognita raça $1 \mathrm{com}$ óleo essencial de Lippia Alba. Scientia Plena, v.8, n.4, p.1-8, 2012.

MATOS, F.J.A. As ervas-cidreiras do Nordeste do Brasil - estudo de três quimiotipos de Lippia alba (Mill.) N. E. Brown (Verbenaceae): Parte I - Farmacologia. Revista Brasileira farmacolgnosia, v.77, n.2, p.65-67, 1996.

McDONALD, D. Some interactions of plant parasitic nematodes and higher plants. In: KRUPA, S.V.; DOMMENGERS, Y.R. (Eds.). Ecology of root pathogens. 1.ed. Amsterdam: Elsevier Scientific Publishing Co., 1979. p.157-281.

MOREIRA, F.J.C. et al. Eclosão e mortalidade de juvenis $\mathrm{J} 2$ de Meloidogyne incognita raça 2 em óleos essenciais. Revista Ciência Agronômica, v.40, n.3, p.441-448,
2009.

MOREIRA, F.J.C.; FERREIRA, A.C.S. Controle alternativo de nematóide das galhas (Meloidogyne enterolobii) com cravo de defunto (Tagetes patula L.), incorporado ao solo. Holos, v.1, p.99-110, 2015.

MULLIN, B.A. et al. Root-knot nematodes associated with beans in Colombia and Peru and related yeld loss. Plant Disease, v.75, p.1208-1211, 1991.

NTALLI, N.G. et al. Synergistic and antagonistic interactions of terpenes against Meloidogyne incógnita and the nematicidal activity of essential oils from seven plants indigenous to Greece. Pest Management Science, v.67, p.341-351, 2011.

OKA, Y. et al. Nematicidal activity of essential oils and theirs components against the root-knot nematode. Phytopathology, v.90, p.710-715, 2000.

OKA, Y. Nematicidal activity of essential oils components against the root-knot nematode Meloidogyne javanica. Nematology, v.3, n.2, p.159-164, 2001.

PARK, I.K. et al. Nematicidal Activity of Plant Essential Oils and Components From Ajowan (Trachyspermum ammi), Allspice (Pimenta dioica) and Litsea (Litsea cubeba) Essential Oils Against Pine Wood Nematode (Bursaphelenchus Xylophilus). Journal of Nematology, v.39, n.3, p.275-279, 2007.

RODRIGUEZ-KABANA, R. et al. Control of plant parasitic nematodes with furfural-a naturally occurring fumigant. Nematropica, v.23, p.63-73, 1993.

SANGWAN, N.K. et al. Nematicidal activity of essential oils of Cymbopogon grasses. Nematologia, v.32, p. 93-99, 1985.

SILVA, S.R.S. et al. Análise de constituintes químicos e da atividade antimicrobiana do óleo essencial de Melaleuca alternifolia Cheel. Revista Brasileira de Plantas Medicinais, v.6, p.63-70, 2003.

SILVEIRA, J.C. et al. Levantamento e análise de métodos de extração de óleos essenciais. Enciclopédia Biosfera, v.8, n.15, p.2038-2051, 2012.

SIMÕES, C.M.O.; SPITZER, V. Óleos voláteis. In: SIMÕES, C.M.O. et al. Farmacognosia: da planta ao medicamento. 1.ed. Porto Alegre: Universidade Federal do Rio Grande do Sul, 1999. p.387-415.

TAVARES, E.S. et al. Análise do óleo essencial de folhas de três quimiotipos de Lippia alba (Mill.) N. E. Br. (Verbenaceae) cultivados em condições semelhantes. Brazilian Journal of Pharmacognosy, v.15, n.1, p.15, 2005.

TSAI, B.Y. et al. Screening plants for nematicidal agents. In: KUBU, I.; JACOBSON, M. (Eds.). Phytochemical Pesticides. v.11, p.1-26, 1991. 